\title{
Behavior of information flow near criticality
}

\author{
Matthijs Meijers, ${ }^{1}$ Sosuke Ito, ${ }^{1,2}$ and Pieter Rein ten Wolde ${ }^{1}$ \\ ${ }^{1}$ NWO Institute AMOLF, 1098 XG Amsterdam, The Netherlands \\ ${ }^{2}$ Universal Biology Institute, The University of Tokyo, 7-3-1 Hongo, Bunkyo-ku, Tokyo, 113-0033, Japan
}

(Received 31 May 2019; revised 18 November 2019; accepted 22 December 2020; published xxxxxxxxxx)

\begin{abstract}
Recent experiments have indicated that many biological systems self-organize near their critical point, which hints at a common design principle. While it has been suggested that information transmission is optimized near the critical point, it remains unclear how information transmission depends on the dynamics of the input signal, the distance over which the information needs to be transmitted, and the distance to the critical point. Here we employ stochastic simulations of a driven two-dimensional Ising system and study the instantaneous mutual information and the information transmission rate between a driven input spin and an output spin. The instantaneous mutual information varies nonmonotonically with the temperature but increases monotonically with the correlation time of the input signal. In contrast, there exists not only an optimal temperature but also an optimal finite input correlation time that maximizes the information transmission rate. This global optimum arises from a fundamental trade-off between the need to maximize the frequency of independent input messages, the necessity to respond fast to changes in the input, and the need to respond reliably to these changes. The optimal temperature lies above the critical point but moves toward it as the distance between the input and output spin is increased.
\end{abstract}

DOI: 10.1103/PhysRevE.00.000100

Most, if not all, living organisms need to respond to changes in their environment. Examples include bacteria searching for food, animals trying to catch prey, or birds in flocks trying to coordinate their motion. In all these cases, the flow of information, be it via an intracellular biochemical network, an intercellular neural network, or between the individuals within the group, is vital to function. Moreover, in all these examples not only the reliability of information transmission is important but also the speed and the distance over which the information needs to be transmitted.

Recent experiments indicate that many biological systems self-organize at their critical point. Examples are the flocking behavior of starlings [1], signal percolation within a bacterial community [2], or neural networks [3]. A hallmark of all these systems is that they process information that is in the dynamics of an input signal-a raptor [1], an electrochemical signal [2], or a neuronal stimulus [3] - by mapping that input onto an output signal-a direction of the birds' course, halting cell growth, or a neuronal response, respectively. To characterize this information transmission capacity [4], we need to understand how these systems process time-varying signals. Moreover, as an information measure we need the information transmission rate [5-7] or the transfer entropy [10], which takes into account both the reliability and the speed of information transmission.

The observation that very different systems self-organize near the critical point hints at a common design principle [11]. The Ising system is, because of its generic properties, ideally suited to address this question. In fact, the role of criticality in information transmission in the Ising system has been studied extensively [12-16]. It has been shown that the mutual information between pairs of neighboring spins exhibits a sharp peak near the critical point $[12,13]$. Additionally, the transfer entropy [10] between pairs of neighboring spins peaks at the critical point while a global transfer entropy measure peaks above it [14]. However, all these studies consider information transfer between spins in Ising systems that are in thermodynamic equilibrium. They do not address the question how the system processes time-varying signals, which, moreover, can drive the system out of thermodynamic equilibrium.

Here we consider a two-dimensional (2D) Ising system that needs to process a time-varying input signal. The input signal $\mathcal{S}$ is a spin, the input spin, which is flipped according to a stationary random telegraph process with a timescale $\tau_{s}$. Its dynamics can thus be regulated independently of the remaining spins, which is controlled by the temperature. The input signal will drive the system out of equilibrium, and because this signal is stationary, our system is in a stationary nonequilibrium steady state. The output signal $\mathcal{X}$ is another spin, the output spin, which is located at a distance $d$ away from the input spin, see Fig. 1. The input and output signals produce the random variables $S, X \in\{+1,-1\}$ at each point in time, respectively. Because the information is propagated from the input to the output spin via the other spins, the dynamics of the output are distinctly non-Markovian [7]. Consequently, we need to recognize the history of the input and output signal in characterizing the information flow between them.

To characterize information flow, not only the mutual information [12,13] and the transfer entropoy [14] have been employed but also various decompositions thereof [15-20]. Yet none of these studies have considered the information 


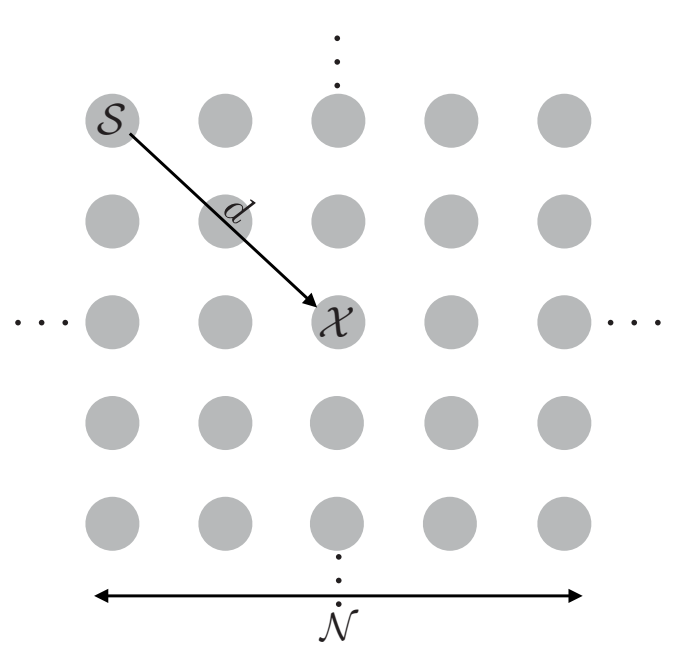

FIG. 1. We consider an Ising system containing $\mathcal{N} \times \mathcal{N}$ spins with periodic boundary conditions. One spin is chosen to be the input spin and is flipped according to a stationary random-telegraph progress. We measure the information transmission from the input to the output spin, positioned a distance $d$ along the diagonal from the input; $d$ in units of the distance between two neighboring spins along the diagonal.

transmission rate or the multistep transfer entropy [10], which take the full history of the input into account. This is critical, because correlations in the input and output signal will affect information transmission.

To characterize information transmission, we study two measures: the instantaneous mutual information $I_{\text {inst }}$ and the information transmission rate $I_{R}$. The measure $I_{\text {inst }}(S ; X)$ is the mutual information between the stationary input and output signal at a single point in time:

$$
I_{\text {inst }}(S ; X)=H(S)-H(S \mid X),
$$

where $H(S)$ is the Shannon entropy of the input signal and $H(S \mid X)$ is the Shannon entropy conditional on the output signal. The instantaneous mutual information has been used to study information transmission in intracellular signaling networks [21-25]. In contrast to the mutual information studied by Matsuda et al. [12], which characterizes the statistical mutual dependence between pairs of spins in an equilibrium system, $I_{\text {inst }}(S ; X)$ quantifies the dependence between the input spin $S$ and the output spin $X$ of our driven system; $2^{I_{\text {inst }}(S ; X)}$ can be interpreted as the number of distinct mappings between input and output that can be distinguished reliably [26]. The quantity $I_{\text {inst }}(S ; X)$ depends on the input timescale $\tau_{s}$ and the response time $\tau_{r}$ of the system, which is determined by the temperature.

While the instantaneous mutual information $I_{\text {inst }}(S ; X)$ quantifies how accurately the input spin is mapped onto the output spin, it does not quantify the rate of information transmission. The latter is determined not only by the accuracy of the input-output mapping but also by the rate at which independent "messages" are transmitted through the system. Autocorrelations within the input and the output signal lower the information transmission rate. To take these correlations into account, we study the information transmission rate, which is defined as the rate at which the mutual information $I\left(\mathbf{S}_{L} ; \mathbf{X}_{L}\right)$ between the trajectories of the input and output signal increases [6]:

$$
I_{R}=\lim _{L \rightarrow \infty} \frac{I\left(\mathbf{S}_{L} ; \mathbf{X}_{L}\right)}{L}=\lim _{L \rightarrow \infty} \frac{H\left(\mathbf{S}_{L}\right)-H\left(\mathbf{S}_{L} \mid \mathbf{X}_{L}\right)}{L},
$$

where $\quad \mathbf{S}_{L}=\left[S\left(t_{1}\right), S\left(t_{2}\right), \ldots, S\left(t_{n}\right)\right] \quad$ and $\quad \mathbf{X}_{L}=$ $\left[X\left(t_{1}\right), X\left(t_{2}\right), \ldots, X\left(t_{n}\right)\right]$ are spin trajectories of duration $L=(n-1) \delta t$, containing $n$ subsequent spin states $S(X)$ at successive time points $t_{i}=(i-1) \delta t$, with $\delta t$ the elementary time step of the dynamics [7]. To capture the autocorrelations in the input and output signal, the trajectory lengths have to be longer than the longest timescale in the problem, $L>\tau_{s}, \tau_{r}$; $I_{R}$ then properly takes into account the history of the input and output spin, in contrast to the one-step transfer entropy. Indeed, in general, $I_{R}$ differs from $I_{\text {inst }} / \delta t$ precisely because of the correlations in input and output. Since in our system the output signal does not feed back on the input, $I_{R}$ is equivalent to the multistep entropy $[5,7]$.

In order to evaluate the effects of the dynamics and criticality on information flow, we will study both measures as a function of the input correlation time $\tau_{s}$, the temperature $T$, the distance $d$ between the input and output spin, and the system size. We are mainly interested in temperatures higher than the critical temperature $T_{c}$, since for lower temperatures the system freezes down in the ferromagnetic phase, drastically slowing down information transmission. We will show that the nontrivial interaction between the diverging correlation length and the diverging response time near the critical point causes the information flow to be optimal close to, but not at, the critical point. The optimal temperature is determined by the distance over which the information needs to be transmitted and the size of the system.

Consider a 2D Ising system of $\mathcal{N} \times \mathcal{N}$ spins with periodic boundary conditions and no external magnetic field. The Hamiltonian of the system, with spin configuration $\sigma=$ $\sigma_{1}, \ldots, \sigma_{\mathcal{N}^{2}}$ and $\sigma_{i} \in\{+1,-1\}$, is $H(\boldsymbol{\sigma})=-J \sum_{\langle i, j\rangle} \sigma_{i} \sigma_{j}$, where $J$ is the coupling parameter and the sum is taken over all nearest neighbors. For isotropic coupling, the critical temperature is $k_{B} T_{c} / J=2.269$ [27]. Following Barnett et al. [14], we use discrete-time Glauber spin-flip dynamics [28]. We define the response time $\tau_{r}$ as the timescale over which spontaneous fluctuations in the undriven system relax to equilibrium, as measured via the two-point time correlation function of the input and output spin [29]. Entropies are measured in nats.

The information transmission rate is notoriously difficult to compute, because the state space of the input and output trajectories rapidly diverges with their length. We have therefore considered not only relatively small systems, but also developed the following scheme: To limit the size of the state space, we introduce a sampling interval $\Delta t$ such that the trajectory length $L=(n-1) \Delta t$. We ensure that $L$ is longer than the input and output correlation time such that $I\left(\mathbf{S}_{L} ; \mathbf{X}_{L}\right)$ increases linearly with $L$ and the information transmission rate $I_{R}$ is independent of $L$ (see Ref. [7]). We then compute, for long-enough $L, I_{R}=I_{R}(\Delta t)$ for a range of $\Delta t$ values, where we verify that the entropy histograms are sampled accurately, using the Bayesian entropy estimator of Nemenman et al. [30]. We then extrapolate $I_{R}(\Delta t)$ to the quantity of interest, 

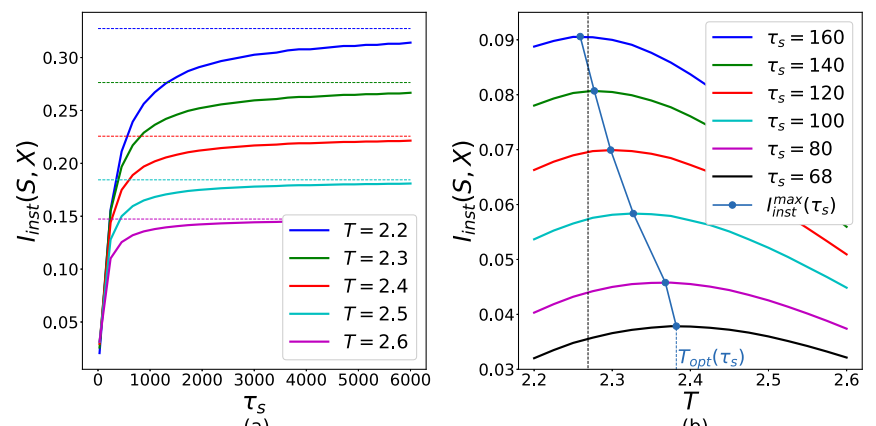

(a)

(b)

FIG. 2. The instantaneous mutual information $I_{\text {inst }}(S ; X)$ as a function of the input correlation time $\tau_{s}$ and temperature $T$. (a) $I_{\text {inst }}(S ; X)$ increases monotonically with $\tau_{s}$ until it reaches a plateau, $I_{\text {inst }, \infty}(S ; X)$, which is equal to the static mutual information (dashed line). The plateau value $I_{\text {inst, } \infty}(S ; X)$ increases as $T$ decreases. However, for small $\tau_{s}$, when $\tau_{s}$ is on the order of the response time $\tau_{r}, I_{\text {inst }}(S ; X)$ does not rise monotonically with decreasing temperature. This is more clearly seen in panel (b): There exist an optimal temperature $T_{\mathrm{opt}}$ that maximizes $I_{\text {inst }}(S ; X)$ for a given $\tau_{s}$; moreover, $T_{\mathrm{opt}}$ decreases when $\tau_{s}$ increases. The optimal temperature arises from a trade-off between responding rapidly and reliably. Vertical dashed line denotes critical temperature. The size of the system is $5 \times 5$, and the distance between input and output spin is $d=2$. Figure S8 of Ref. [7] shows that the results do not qualitatively change for a $10 \times 10$ times larger system.
$I_{R}(\Delta t \rightarrow \delta t)$, where $\delta t$ is the elementary time step of the Glauber dynamics; to verify this extrapolation procedure, we have also recomputed $I_{R}(\Delta t)$ for a number of extrapolated $\Delta t$ values (see Ref. [7]).

Figure 2 shows the instantaneous mutual information $I_{\text {inst }}(S ; X)$ between the input and output signal separated by a distance $d=2$ as a function of the input correlation time $\tau_{\mathrm{s}}$ and temperature $T$ in an Ising system of $5 \times 5$ spins. The instantaneous mutual information rises with the input correlation time $\tau_{s}$ [Fig. 2(a)], because this gives the system more time to respond to changes in the input signal and hence more time to correlate the output with the input signal. For large $\tau_{s} \rightarrow \infty$, the instantaneous mutual information reaches a plateau value $I_{\text {inst, } \infty}(S ; X)$ that corresponds to the static mutual information, which is the mutual information between the output spin and the input spin when the latter is held fixed indefinitely for each realization $S=1,-1$. The static mutual information increases as the temperature is decreased, because decreasing the temperature lowers the thermal noise in the transmitted signal.

Figure 2(b) shows that for a given correlation time $\tau_{s}$ of the input signal, there exists an optimal temperature $T_{\mathrm{opt}}$ that maximizes the instantaneous mutual information $I_{\text {inst }}(S ; X)$. Increasing the temperature raises the thermal noise in the signal, which tends to lower the instantaneous mutual information. On the other hand, increasing the temperature also reduces the response time $\tau_{r}$. This allows the system to more accurately track the input signal, which tends to raise the instantaneous mutual information between the input and output signal. The interplay between these two effects gives rise to an optimal temperature $T_{\mathrm{opt}}\left(\tau_{s}\right)$ that maximizes the instantaneous mutual information, $I_{\text {inst }}^{\max }\left(\tau_{s}\right)$. This optimal temperature

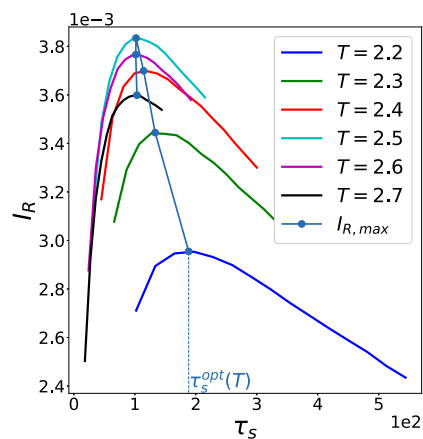

(a)

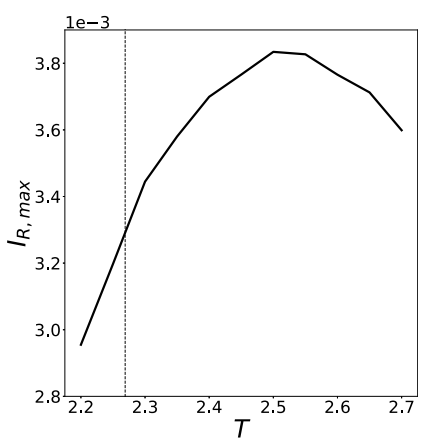

(b)
FIG. 3. The information transmission rate $I_{R}$ as a function of the input correlation time $\tau_{s}$ and temperature $T$. (a) For a given temperature $T$, there exists an optimal $\tau_{s}$ that maximizes the information transmission rate $I_{R}, I_{R}^{\max }$; it arises from a trade-off between maximizing the frequency of independent inputs and responding reliably. The figure also shows that $I_{R}^{\max }$ initially rises with $T$, but then decreases, which is highlighted in panel (b): There exists an optimal temperature $T_{\text {opt }}$ that maximizes $I_{R}^{\max }$. Vertical dashed line denotes critical temperature. System size is $5 \times 5$, and the distance between input and output spin is $d=2$. Lines are truncated at high $\tau_{s}$ for large $T$, because it becomes exceedingly difficult to get good statistics in this regime.

decreases as the input correlation time $\tau_{s}$ is increased, because the latter gives the system more time to respond to the changes in the input. Moreover, the maximum instantaneous mutual information $I_{\text {inst }}^{\max }\left(\tau_{s}\right)$ rises with $\tau_{s}$, not only because increasing $\tau_{s}$ raises $I_{\text {inst }}$ by itself, but also because the lower optimal temperature $T_{\mathrm{opt}}\left(\tau_{s}\right)$ reduces the thermal noise in the signal.

Figure 3(a) shows the information transmission rate $I_{R}$ as a function of the correlation time of the input signal $\tau_{\mathrm{s}}$ for different temperatures $T$. While, for a given temperature, the instantaneous mutual information $I_{\text {inst }}$ increases monotonically with the input correlation time $\tau_{s}$ [see Fig. 2(a)], the information transmission rate $I_{R}$ exhibits an optimal $\tau_{s}$ that maximizes $I_{R}$. When $\tau_{s}$ is too short, the signal is changing faster than the output can respond to, which decreases $I_{R}$ by increasing the conditional entropy $H\left(\mathbf{S}_{L} \mid \mathbf{X}_{L}\right)$ [see Eq. (2)]. On the other hand, for large $\tau_{s}$ time is wasted when the output has been correlated to the input yet is waiting for the signal to change again; indeed, the entropy of the input signal $H\left(\mathbf{S}_{L}\right)$ decreases as $\tau_{s}$ is increased, which tends to lower $I_{R}$ [see Eq. (2)]. This interplay causes the information transmission rate to have a maximum at an optimal input timescale $\tau_{\mathrm{s}}^{\mathrm{opt}}$. The value of $\tau_{s}^{\text {opt }}$ decreases with temperature, because at higher temperatures the system can respond more rapidly to changes in the input signal.

Figure 3(b) shows the maximum value of the information transmission rate $I_{R}$ at the optimal input correlation time $\tau_{s}^{\mathrm{opt}}, I_{R}^{\max } \equiv I_{R}\left(\tau_{s}=\tau_{s}^{\mathrm{opt}}\right)$, as a function of the temperature $T$. Clearly, there exists an optimal temperature that maximizes $I_{R}^{\max }$. This is in marked contrast to the maximum value of the instantaneous mutual information, obtained for $\tau_{s} \rightarrow \infty$, $I_{\text {inst, } \infty}$, which increases monotonically with decreasing temperature [see Fig. 2(a)]. The optimum in $I_{R}^{\max }$ arises from the trade-off between a faster response at higher temperatures, which allows for a more rapidly varying input that increases 


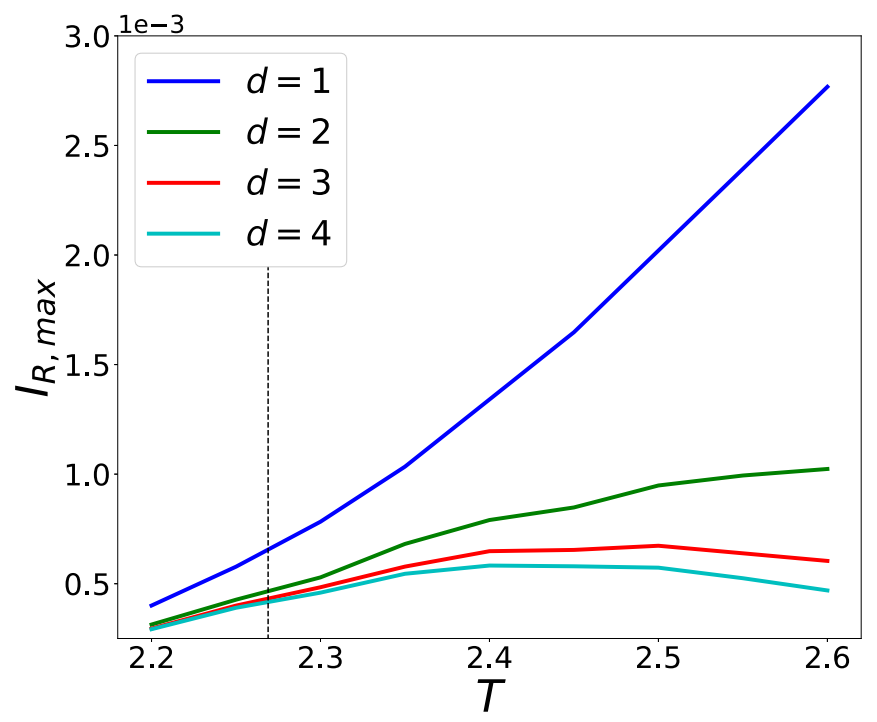

FIG. 4. The optimal temperature that maximizes the information transmission rate $I_{R}$ decreases as the distance $d$ over which the information is transmitted increases. The figure shows for different values of $d$ the maximum value of $I_{R}, I_{R}^{\max }$, obtained by optimizing $I_{R}$ over the input correlation time $\tau_{s}$ [see Fig. 3(a)], as a function of the temperature $T$. It is seen that $I_{R}^{\max }$ decreases as $d$ is increased, while the optimal temperature moves closer to the critical temperature, denoted by vertical dashed line. The system size is $10 \times 10$.
$H\left(\mathbf{S}_{L}\right)$, and less thermal noise in the transmitted signal at lower temperatures, which reduces $H\left(\mathbf{S}_{L} \mid \mathbf{X}_{L}\right)$ [Eq. (2)]. The reason $I_{R}^{\max }$ peaks above the critical temperature $T_{c}$ is that the response time rapidly increases near $T_{c}$, thereby decreasing the amount of information that can be sent through the system per unit amount of time.

So far we have kept both the distance $d$ between the input and output spin constant, as well as the system size. We now systematically vary these parameters. Figure 4 shows the maximum information transmission rate $I_{R}^{\max }$, obtained by optimizing over $\tau_{s}$ (see Fig. 3), as a function of temperature $T$ for different values of $d$ in a $10 \times 10$ Ising system. The transmission rate decreases as $d$ is increased, because the correlations between spins become weaker as the distance between them becomes larger. More interestingly, the optimal temperature that maximizes $I_{R}^{\max }$ moves closer to the critical temperature when $d$ is increased. When the distance $d$ between the input and output spin is increased, the correlation length must be increased in order to maintain the correlations between them. This can be achieved by bringing the system closer to the critical point.

Critical effects are stronger in systems of larger size. Close to $T_{c}$, the response time of our system increases up to sixfold when the system size is increased from $5 \times 5$ to $10 \times 10$ spins. This makes it beneficial for information transmission to move the system further away from the critical point when the system size is increased at constant $d$. Compare the case of $d=2$ in the $5 \times 5$ system in Fig. 3 to that of $d=2$ in the $10 \times 10$ system in Fig. 4: While $I_{R}^{\max }$ decreases because of the larger response time in the larger system, the optimal temperature that maximizes $I_{R}^{\max }$ increases from $T_{\mathrm{opt}} \approx 2.5$ to $T_{\mathrm{opt}} \approx 2.6$ to mitigate the effect of the larger response time, which tends to reduce the information transmisison rate.

The increase in the correlation length and the correlation time when the system is moved toward the critical temperature have opposite effects on information transmission. Moreover, these effects are more pronounced for larger systems, diverging in the thermodynamic limit. These two observations explain, as described above, why the optimal temperature $T_{\mathrm{opt}}$ that maximizes the information transmission rate moves toward the critical temperature $T_{c}$ when the distance $d$ between the input and output spin is increased in a system of constant size (Fig. 4), yet away from it when the system size is increased at constant $d$ (cf. Figs. 3 and 4). This raises the question how $T_{\text {opt }}$ changes as $d$ is scaled together with the system size, which, as renormalization group theory indicates, is also the relevant finite-size scaling question for this problem. We have therefore also performed simulations for $d=6$ and $\mathcal{N}=15$. The optimal temperature that maximizes information transmission decreases from $T_{\text {opt }} \approx 2.53$ for $(d=2, \mathcal{N}=5)$, to $T_{\mathrm{opt}} \approx 2.44$ for $(d=4, \mathcal{N}=10)$, and $T_{\mathrm{opt}} \approx 2.38$ for $(d=6, \mathcal{N}=15)$ (see Fig. S10 [7]). We emphasize that these system sizes are small, but computing $I_{R}^{\max }$ becomes rapidly harder for larger systems. Nonetheless, our results do hint that $T_{\mathrm{opt}}$ moves toward $T_{c}$ in the thermodynamic limit. In the SI, we discuss this question further; here we also study another quantity, the lagged mutual information [7].

In summary, the information transmission rate $I_{R}$ is a dynamic quantity that depends on the input correlation time $\tau_{c}$, the response time $\tau_{r}$, and the reliability of the response. Increasing $\tau_{c}$ gives the system more time to respond to changes in the input, which tends to enhance the reliability of the response, yet also reduces the frequency of independent input messages, which decreases $I_{R}$. Increasing the temperature decreases $\tau_{r}$, which enhances the rate at which information can be transmitted, yet also increases the thermal noise, which reduces the reliability of the response. The information transmission rate thus exhibits an optimal input correlation time and an optimal temperature, which arise from a fundamental trade-off between the need to maximize the input frequency, the necessity to respond fast to changes in the input, and the need to respond reliably to these changes. The optimal temperature depends on the distance between the in- and output spin and the size of the system. The optimal temperature is close to yet above the critical point, although our results hint it moves toward the critical temperature in the thermodynamic limit.

Our results may help to explain why a number of biological systems appear to be tuned close to a critical point [1-3,31] and, more generally, will be relevant for understanding information transmission in these systems. For example, our results predict that increasing the interaction strength between the components of a system, be it between proteins in a biochemical network (between, e.g., the receptor and the kinase CheA in the bacterial chemotaxis system [32]), cells in a bacterial community, or birds in a flock, will on the one hand increase the reliability of information transmission, yet on the other hand also increase the response time of the system, giving rise to an optimal strength that maximizes the information transmission rate. In fact, these principles likely 
pertain to systems outside the biological realm. Last, many systems, including biological systems, are high dimensional. Since the response time does not depend on the dimensionality of the system while correlations decay faster with distance in higher dimension, we conjecture that in higher-dimensional systems the optimal temperature is closer to the critical point.
This work is part of the research programme of the Netherlands Organisation for Scientific Research (NWO) and was performed at AMOLF. Ito was funded by KAKENHI Grant No. JP16K17780 and JST Presto Grant No. JP18070368, Japan. We thank I. Nemenman for sharing his code to estimate entropies and T. Ouldridge for carefully reading our manuscript.
[1] A. Cavagna, A. Cimarelli, I. Giardina, G. Parisi, R. Santagati, F. Stefanini, and M. Viale, Proc. Natl. Acad. Sci. USA 107, 11865 (2010).

[2] J. W. Larkin, X. Zhai, K. Kikuchi, S. E. Redford, A. Prindle, J. Liu, S. Greenfield, A. M. Walczak, J. Garcia-Ojalvo, A. Mugler et al., Cell Syst. 7, 137 (2018).

[3] G. Tkacik, T. Mora, O. Marre, D. Amodei, M. J. Berry, and W. Bialek, Proc. Natl. Acad. Sci. USA 112, 11508 (2015).

[4] C. E. Shannon, Bell Syst. Tech. J. 27, 379 (1948).

[5] J. L. Massey, Proceedings of the 1990 International Symposium on Information Theory and its Applications (ISITA-90) (1990) pp. 303-305.

[6] F. Tostevin and P. Rein Ten Wolde, Phys. Rev. Lett. 102, 218101 (2009).

[7] See Supplemental Material at http://link.aps.org/supplemental/ 10.1103/PhysRevE.xx.xxxxxx for an overview of different information theoretic measures, description of the inference procedure, and additional figures, which includes references $[[8,9]]$.

[8] P. Grassberger, arXiv:physics/0307138.

[9] R.G. Miller, Biometrika 61, 1 (1974).

[10] T. Schreiber, Phys. Rev. Lett. 85, 461 (2000).

[11] T. Mora and W. Bialek, J. Stat. Phys. 144, 268 (2011).

[12] H. Matsuda, K. Kudo, R. Nakamura, O. Yamakawa, and T. Murata, Int. J. Theor. Phys. 35, 839 (1996).

[13] S.-J. Gu, C.-P. Sun, and H.-Q. Lin, J. Phys. A: Math. Theor. 41 (2008).

[14] L. Barnett, J. T. Lizier, M. Harré, A. K. Seth, and T. Bossomaier, Phys. Rev. Lett. 111, 177203 (2013).

[15] V. S. Vijayaraghavan, R. G. James, and J. P. Crutchfield, Entropy 19, 214 (2017).
[16] D. Marinazzo, L. Angelini, M. Pellicoro, and S. Stramaglia, Phys. Rev. E 99, 040101(R) (2019).

[17] J. T. Lizier, F. M. Atay, and J. Jost, Phys. Rev. E 86, 026110 (2012).

[18] M. Wibral, J. T. Lizier, S. Voegler, V. Priesemann, and R. Galuske, Front. Neuroinf. 8, 1 (2014).

[19] L. Faes, A. Porta, G. Nollo, and M. Javorka, Entropy 19, 5 (2017).

[20] A. Kolchinsky and B. Corominas-Murtra, J. R. Soc. Interface 17 (2020).

[21] F. Tostevin and P. R. ten Wolde, Phys. Rev. E 81, 061917 (2010).

[22] R. Brittain, N. Jones, and T. Ouldridge, J. Stat. Mech. (2017) 063502.

[23] S. G. Das, M. Rao, and G. Iyengar, Phys. Rev. E 95, 062410 (2017).

[24] G. Malaguti and P. R. ten Wolde, arXiv:1902.09332v1.

[25] N. Becker, A. Mugler and P. R. Ten Wolde, Phys. Rev. Lett. 115, 258103 (2015).

[26] G. Tkačik and A. M. Walczak, J. Phys. Condens. Matter 23, $153102(2011)$.

[27] L. Onsager, Phys. Rev. 65, 117 (1944).

[28] R. J. Glauber, J. Math. Phys. 4, 294 (1963).

[29] D. Chandler, Introduction to Modern Statistical Mechanics, 1st ed. (Oxford University Press, Oxford, 1987).

[30] I. Nemenman, W. Bialek, and R. de Ruyter van Steveninck, Phys. Rev. E 69, 056111 (2004).

[31] A. Gelblum, I. Pinkoviezky, E. Fonio, A. Ghosh, N. Gov, and O. Feinerman, Nat. Commun. 6, 7729, (2018).

[32] D. Bray, M. D. Levin, and C. J. Morton-Firth, Nature 393, 85 (1998). 